\title{
Como as cidades podem favorecer ou dificultar a promoção da saúde de seus moradores?
}

\author{
LAÍS FAJERSZTAJN I, MARIANA VERAS II \\ e PAULO HILÁRIO NASCIMENTO SALDIVA III
}

\section{Introdução}

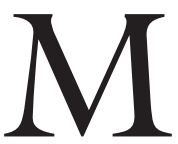

AIS DA METADE da população mundial vive em cidades. Na América Latina - o continente mais urbanizado do mundo -, a população urbana chega a $82 \%$; no Brasil, a $84 \%$. Com tantas pessoas morando em cidades, entender a influência do ambiente construído na saúde das pessoas torna-se assunto prioritário.

Por muitos séculos, o estilo de vida das cidades causou piores condições de saúde, como maior risco para cólera, diarreia, tuberculose e outras doenças infecciosas. A densidade populacional favorecia a emergência de epidemias, e viver em cidades representava uma desvantagem para a saúde em comparação à vida no campo. Com o desenvolvimento científico sobre as formas de transmissão de doenças, iniciado no século XIX, as cidades começaram a se estruturar visando à promoção da saúde pública, principalmente a partir da adoção de medidas sanitárias. Os serviços de assistência à saúde acompanharam o desenvolvimento científico-tecnológico de forma muito mais eficiente nos centros urbanos e o viver nas cidades passou a ser uma vantagem para a saúde, quando comparado com a vida rural.

Hoje doenças diretamente relacionadas à forma como a sociedade está organizada e ao estilo de vida urbano, como hipertensão arterial, diabetes tipo 2 e obesidade, predominam nas cidades. Conhecidas como Doenças e Agravos Não Transmissíveis (Dant), essas doenças foram responsáveis por $76 \%$ da mortalidade geral, 63\% das internações pagas e 73\% dos gastos do Sistema Único de Saúde (SUS) com internações no município de São Paulo, segundo dados do site oficial da prefeitura. A estratégia de enfrentamento tem se baseado principalmente na modificação de fatores de risco individuais, com resultados positivos, porém limitados, visto que o ambiente urbano pode facilitar ou dificultar hábitos individuais saudáveis, como a prática de atividade física ou a ingestão de frutas e vegetais. Sendo assim, a integração de políticas de promoção da saúde coletiva 
e de urbanismo resultaria em ganhos de saúde significativos para os moradores de cidades.

Alguns exemplos bem-sucedidos de controle e prevenção de doenças a partir da modificação de fatores de risco individuais incluem o controle medicamentoso da pressão arterial e colesterol, parar de fumar, alimentar-se de forma mais saudável e praticar atividade física. A lei antifumo, que proibiu fumar em ambientes fechados de uso coletivo no estado de São Paulo em 2009, diminuiu a exposição dos não fumantes à fumaça do tabaco. Tal lei ainda resultou na diminuição da quantidade de cigarros/dia entre os fumantes, sendo um exemplo concreto de como o funcionamento de uma cidade pode melhorar a saúde de sua população. Com custo anual estimado em mais de $\mathrm{R} \$ 40$ bilhões, valor equivalente a $1 \%$ do PIB brasileiro (Cintra, 2014), a imobilidade no município de São Paulo é um exemplo oposto. A crise de mobilidade afeta a saúde não só por conta do tempo perdido no congestionamento e impactos nocivos da exposição à poluição do ar à saúde, em certa medida estimados no cálculo, mas também pela sua contribuição para a obesidade, estresse emocional, entre outros. Numa cidade congestionada, crianças não brincam na rua e adultos não retornam para almoçar em casa, costume prevalente em São Paulo quando a obesidade ainda não era uma epidemia.

Ademais, o progresso que resultou na vantagem para a saúde de viver nas cidades não atingiu todos os moradores de países em desenvolvimento. Ainda hoje, populações urbanas de baixa renda, particularmente residentes de favelas, ainda convivem com a falta de saneamento básico e condições seguras de moradia. Frequentemente essas populações sofrem com enchentes, falta de água, surtos de doenças infecciosas, entre outros riscos para a saúde, cujas formas preventivas são amplamente evitáveis e conhecidas. Além disso, determinantes sociais como escolaridade, moradia, alimentação e renda exercem influência negativa em moradores de favela, prejudicando ainda mais o desempenho em saúde dessa parcela da população. Por fim, a violência urbana diminui o desempenho da saúde de toda a população, seja causando mortes e incapacidades diretamente, seja prejudicando a saúde mental, ou ainda, restringindo a mobilidade e lazer dentro das cidades. A Região Metropolitana de São Paulo (RMSP) lidera a prevalência de desordens mentais no mundo, quase $30 \%$ da população apresentam algum distúrbio como ansiedade e abuso de substâncias, segundo estudo realizado por Andrade et al. (2012). Os pesquisadores mostraram que a exposição à violência (como ter presenciado um assassinato ou ter sido roubado) estava significativamente associada com as desordens mentais e, quanto maior a exposição à violência, a gravidade da doença também tendia a ser maior (Andrade et al., 2012).

Durante as últimas décadas, a Organização Mundial da Saúde (OMS) tem tentado fortalecer as relações entre saúde e planejamento urbano por meio de dois movimentos: Saúde em todas as políticas (Health in all polices) (Ollila, 
2011) e Movimento das cidades saudáveis (Healthy cities movement) (Rydin et al., 2012); no entanto, esses movimentos ainda não são fortes nas cidades latino-americanas.

Recentemente, a preocupação da comunidade de saúde com os impactos das mudanças climáticas tem crescido, sendo outra abordagem possível para fortalecer a comunicação entre ambiente construído e melhor desempenho de indicadores de saúde. Em 2009, uma série de artigos publicados no The Lancet indicou que algumas ações que reduzem as emissões de gases de efeito estufa trazem também benefícios para a saúde da população onde as medidas foram adotadas (Haines et al., 2009). Em 2015, a mesma revista alertou para o fato de que combater as mudanças climáticas pode ser a melhor oportunidade de saúde do século XXI (Dora et al., 2015; Watts et al., 2015). Como as populações de baixa renda são mais vulneráveis aos impactos das mudanças climáticas em todas as escalas (entre nações e entre cidades), combater as mudanças climáticas pode ser uma oportunidade para reduzir as desigualdades de saúde.

O desafio de moldar cidades que promovam a saúde de seus moradores é complexo e requer esforço multidisciplinar para muito além das competências atuais atribuídas ao setor de saúde no Brasil. O experimento "Moving to Oportunity" conduzido pela universidade de Harvard com 4.600 famílias mostrou que prover assistência para que famílias de baixa renda se mudem para bairros mais saudáveis melhorou significativamente a saúde física e mental de crianças e adultos (Chetty et al., 2015).

Neste artigo propomos uma discussão das relações entre o ambiente construído e a saúde coletiva a partir dos cinco tópicos propostos por Rydin et al. (2012): a) transporte, mobilidade e atividade física; b) produção urbana de alimento; c) ilhas urbanas de calor; d) conforto térmico e qualidade do ar de ambientes fechados (indoor); e e) saneamento.

\section{a) Transporte, mobilidade e atividade física}

O custo anual da imobilidade na cidade de São Paulo equivale a 7,5\% do PIB da cidade (Cintra, 2014), com impactos significativos para a saúde dos moradores. Os veículos automotores são os principais responsáveis pelos altos índices de poluição do ar aos quais os paulistanos estão expostos. Estima-se que a poluição do ar em São Paulo reduza em cerca de 3,5 anos a expectativa de vida média desta população.

O meio de transporte utilizado por um indivíduo em seu dia a dia pode ser determinante para sua saúde. De fato, transporte é um dos determinantes sociais da saúde, segundo a Organização Mundial da Saúde (OMS). Além disso, as emissões veiculares que contribuem tanto para a poluição do ar urbano quanto para as alterações no clima global também impactam diretamente de forma negativa a saúde das pessoas (WHO, 2011).

Por isso, tanto o planejamento quanto as políticas públicas de transporte devem considerar a saúde como um dos critérios de avaliação durante a formu- 
lação e mesmo após sua implementação. Na América Latina a inclusão da saúde como um determinante durante esse processo não parece ser uma rotina.

A urbanização latino-americana é caracterizada pela industrialização, pela deterioração ambiental, cidades superpopulosas cercadas por uma periferia pobre, congestionamentos e poucas opções eficientes de transporte público. E o transporte individual em veículos automotores é o modo de transporte prevalente. Contribui para essa escolha o fato de "ter um carro" ser sinônimo de mobilidade, conforto e status social (Newman; Kenworthy, 1999).

Olhando sob a perspectiva da saúde, os carros são prejudiciais, pois diminuem a atividade física, contribuem para o isolamento social, causam estresse e a perda de horas de descanso, lazer e trabalho, devido aos congestionamentos. Além disso, colaboram significativamente para a poluição do ar e sonora, e acidentes por automóveis provocam a morte de milhares de pessoas todos os anos (apud McMichael, 1996; Rissel, 2009). A poluição do ar tem efeitos agudos e crônicos reconhecidos. Além do desconforto geral (ardor nos olhos, alergias etc.), a exposição de curto período a altos níveis de poluentes atmosféricos aumenta a internação hospitalar por doenças cardiovasculares e respiratórias (Braga et al., 2002, Saldiva et al., 1992). Entre 1996 e 2000 , o aumento de $10 \mu \mathrm{g} / \mathrm{m}^{3}$ do poluente MPl0 (material particulado menor que 10 micrometros) associou-se com um aumento nas admissões hospitalares de $4,6 \%$ por asma em crianças e $4,3 \%$ por doença pulmonar obstrutiva crônica (Gouveia et al., 2006). A exposição prolongada à poluição do ar aumenta o risco de câncer pulmão (Loomis et al., 2013) e de morte por causas cardiovasculares e respiratórias. Baixo nível socioeconômico e faixa etária (fetos, crianças e idosos) apresentam maior vulnerabilidade, assim como ser portador de doenças crônicas ou estar grávida. A exposição à poluição do ar está associada com hipertensão gestacional, baixo peso ao nascer e prematuridade (Stieb et al., 2012). O sistema endócrino (diabetes e obesidade) e o neurocognitivo, entre outros, também são negativamente afetados pela poluição do ar.

A saúde pode ser impactada diretamente de forma positiva pela escolha do meio de transporte que utilizarmos, prevenindo doenças ligadas ao sedentarismo e, indiretamente, pela diminuição dos efeitos causados ao meio ambiente devido às emissões dos escapamentos. As alternativas de transporte benéficas à saúde incluem caminhar, andar de bicicleta e utilizar o transporte público. Entretanto, para que as pessoas utilizem meios mais ativos de transporte, seria necessário que esses oferecessem conforto, segurança, acessibilidade, baixo custo e, principalmente, que fossem integrados de forma a estimular seu uso.

Um dos maiores desafios dos planejadores urbanos é melhorar o transporte urbano em termos de eficiência no deslocamento e diminuição das emissões veiculares. Uma forma de estimular o uso do transporte ativo é integrar ciclovias ao sistema de transporte público. Isso já é empregado na Europa e em alguns 
países na América Latina, com excelentes resultados na saúde da população e para o meio ambiente (Pucher; Buehler, 2008; 2007; Gomez et al., 2015).

Um revisão sistemática recente sobre benefício do transporte ativo para a saúde mostra claramente que essa opção gera grandes ganhos apesar dos riscos de acidentes e da exposição à poluição do ar. Os benefícios estão ligados ao fato de o transporte ativo proporcionar um aumento na atividade física e interação social, reduzindo os riscos para doenças como diabetes, hipertensão e obesidade (Muller et al., 2015). E essa é uma questão muito importante de saúde pública, pois $30 \%$ dos adultos fazem menos exercício que o recomendado, o que juntamente com uma dieta rica em calorias e gorduras é a principal causa da obesidade.

$\mathrm{Na}$ América Latina, ao contrário do que ocorre na Europa e na América do Norte, o apelo para mudanças nas políticas de transporte está relacionado à resolução de problemas de mobilidade (congestionamentos) e os argumentos são praticamente baseados somente em questões econômicas e materiais (Becerra et al., 2013). Os setores da saúde e do do meio ambiente deveriam participar do processo de tomada de decisão sobre mobilidade e os planejadores urbanos deveriam estar conscientes dos impactos que o ambiente construído e dos meios de transporte trazem à saúde física e mental. As iniciativas de estímulo e implementação de alternativas de transporte ativo ou sua integração ao sistema tradicional na América Latina são poucas. Mas em algumas grandes cidades, como Rio de Janeiro, São Paulo, Curitiba, Bogotá, Buenos Aires e Santiago, já se observam mudanças e a implementação de ciclovias integradas como opção alternativas ao transporte individual. Também há em grandes capitais a implantação dos BRT, que são ônibus de trânsito rápido em vias exclusivas (México, São Paulo, Belo Horizonte, Equador). Essas medidas reduzem o número de veículos na rua e, portanto, os níveis de poluentes tendem a cair, e dessa forma também contribuem para uma melhora na saúde geral da população.

O estudo de Laverty et al. (2015) examinou a correlação entre dados sociais e demográficos e o uso de meios de transporte ativo e desfechos específicos de saúde (diabetes, pressão arterial, sobrepeso) de adultos em seis países (México, Índia, China, Rússia, Gana e África do Sul). Os seus resultados mostraram que os indivíduos que realizam seus deslocamentos de forma mais ativa apresentam riscos reduzidos para ganho de sobrepeso e alterações prejudiciais na pressão arterial.

A poluição do ar é o principal fator de risco ambiental para a saúde e o único meio de evitá-lo é reduzindo seus níveis, ou seja, reduzir as emissões. A OMS estima que 1,3 milhão de mortes por ano estejam associadas a exposição à poluição do ar no mundo. Os impactos sobre a saúde incluem uma grande variedade de efeitos que são influenciados por idade, sexo e presença de doenças preexistentes no indivíduo exposto: doenças cardiorrespiratórias, doenças neurodegenerativas, diminuição da fertilidade e câncer (HEI, 2010; Calderón- 
-Garcidueñas et al., 2008). A frota de veículos também contribui para a emissão de gases do efeito estufa colaborando de forma efetiva para acelerar as mudanças climáticas e seus impactos sobre a saúde. Temperaturas extremas estão associadas a um aumento de risco para mortalidade por diferentes causas (Anderson; Bell, 2009; Analitis et al., 2008). Um estudo recente de nosso grupo de pesquisa mostrou que variações moderadas na temperatura também estão associadas a riscos aumentados para mortalidade reforçando a importância das alterações climáticas (variações térmicas) na saúde (Gasparrini et al., 2015).

O transporte ativo integrado a um sistema de transporte público eficiente é uma das alternativas para melhorar a saúde humana, reduzir a poluição ambiental e mitigar as mudanças climáticas. O desafio que os planejadores urbanos e formuladores de políticas enfrentam hoje no desenvolvimento de municípios é como implementar isso em um ambiente urbano concebido para favorecer o modo de transporte privado. Em suma, o transporte ativo não é apenas um meio de reduzir a poluição do ar e as mudanças climáticas em médio e longo prazos, mas também uma forma de melhorar a saúde de milhões de pessoas em curto prazo.

\section{b) Produção urbana de alimento}

A produção de alimentos em ambientes urbanos não é uma novidade (FAO, 2014). Insegurança alimentar devido à pobreza (Zezza; Tasciotti, 2010; Wolch et al., 2014), guerra (Milbourn; Vail, 2010, Rydin et al., 2012) e desastres naturais (Hara et al., 2013) garantiu a produção urbana de alimentos nas últimas décadas, mesmo que em pequena escala. Com apelos fundados na sustentabilidade ambiental, atualmente a UPA (sigla em inglês para agricultura urbana e periurbana) tem ressurgido nas cidades mais sustentáveis dos países desenvolvidos (Midle et al., 2014; Hara et al., 2013; Rydin et al., 2012). Ao invés de substituir as atuais e eficientes formas de produção de alimentos para suprimento das cidades, a proposta da UPA é conviver com as opções tradicionais. Segundo relatório da Organização das Nações Unidas para a Alimentação e Agricultura (FAO) de 2014, a UPA está presente em muitas cidades da América Latina, principalmente em quintais residenciais e escolas, mas também de forma comercial. Rosario, uma cidade de 1,35 milhão de habitantes na Argentina, tem 800 hortas comunitárias que produzem verduras para 40 mil pessoas. Cerca de 23 mil hectares de terra são dedicados à plantação na cidade do México, e em Cuba, 40 mil pessoas trabalham em hortas urbanas. Os benefícios da agricultura urbana incluem melhorias ambientais, econômicas, sociais e de saúde.

Comparado com outros residentes urbanos, famílias de agricultores consomem mais frutas e vegetais. A baixa ingestão de frutas e vegetais é um dos dez fatores de risco que mais contribuem para a sobrecarga da doença (Lim et al., 2012). Alimentação de qualidade melhora a saúde cardiovascular (Hu et al., 2011 ) e ajuda a combater outras doenças crônicas não transmissíveis como obesidade (Gatto et al., 2015) e diabetes (Tohill et al., 2004). A UPA cria oportu- 
nidades para a prática de atividade física e interação social reforçando os ganhos em saúde já descritos (Rydin et al., 2012).

Os serviços ecológicos da agricultura urbana podem incluir a regulação do microclima local, manutenção da fertilidade do solo e regulação da polinização dependendo da forma como é conduzida, entre outros (Swinton et al., 2007). Quando o alimento é produzido localmente, a demanda por transporte é reduzida, resultando em economia de energia e diminuição na emissão de poluentes atmosféricos. Outros ganhos em potencial incluem a redução de exportação de resíduos sólidos, desde que projetos de compostagem para uso na UPA sejam implementados, e melhor manejo da água de chuva. Isso porque a maior prevalência da UPA aumenta a superfície de cobertura verde permeável na cidade, permitindo a recuperação das reservas de água (Angnotti, 2015) e auxiliando a prevenção de inundações durante períodos chuvosos. Vale ressaltar ainda que o aumento da cobertura verde urbana tem sido relacionado a diversos benefícios à saúde mental.

A UPA também tem potenciais ganhos econômicos diretos. Famílias envolvidas com a UPA diminuem seus gastos com comida e geram renda extra com vendas, caso esta não seja a atividade principal da família (FAO, 2014). Revitalização de comunidades de baixa renda (Angnotti, 2015) e aumento no valor dos imóveis da região da UPA pela melhoria estética da região (Rydin et al., 2012) podem emergir como ganhos sociais e econômicos indiretos.

Os desafios técnicos com a qualidade das sementes, solo, entre outros, são frequentes, mas podem ser superados. O Programa Escola Estufa Lucy Montoro de 2009, que, entre outros, visa estimular a produção de hortaliças e mudas de árvores é um exemplo de apoio da prefeitura de São Paulo para a atividade. Abordagens mais ecológicas de produção podem contribuir para a preservação do meio ambiente como a poluição das águas e para a redução de doenças ocupacionais por exposição a agrotóxicos.

O maior desafio da UPA é o espaço. Entretanto, estratégias urbanísticas, como telhados verdes (Torrenggiani et al., 2012), podem viabilizar a UPA mesmo em cidades altamente populosas.

Por fim, é importante atentar para a alta prevalência de solos contaminados em áreas urbanas. O estudo dos impactos para a saúde de alimentos produzidos em solos contaminados é escasso. Antes de escolher uma área para UPA, é recomendada uma avaliação das condições locais (solo, incidência de luz, oferta de água etc.). A história de ocupação do solo local pode indicar de forma indireta o potencial de contaminação do solo, na ausência de testes de contaminação (padrão de referência). No caso de atividades industriais, postos de gasolina e lavanderias é prudente considerar o solo contaminado. A remediação é frequentemente possível, mas a falta e alto custo de serviços especializados podem ser um impeditivo. Uma alternativa possível é importar solo saudável e plantar em vasos (Rydin et al., 2012; Angnotti, 2015; Kessler, 2013). Para prevenir exposi- 
ções nocivas é prudente seguir recomendações de cultivo como as propostas por Kessler (2013) para agricultores urbanos. As recomendações incluem práticas de agricultura (exemplo: cobrir o solo e as passagens existentes com adubo), bons hábitos (exemplo: usar luvas) e critérios de escolha do local da atividade (exemplo: estabelecer a atividade longe de ruas e avenidas).

Ante os potenciais benefícios para a saúde ambiental e humana discutidos, a OMS recomendou que políticas alimentares sejam incorporadas no planejamento urbano (Rydin et al., 2012).

\section{c) Ilhas urbanas de calor}

Ilha urbana de calor é a terminologia que caracteriza o aumento de temperatura de áreas urbanas, comparado à temperatura prévia na mesma região em anos anteriores, ou em regiões do entorno durante o mesmo período. Consequência do processo de urbanização, a substituição da cobertura vegetal do solo por prédios, ruas, avenidas e outras construções de infraestrutura absorve a luz solar, irradia calor e retém menos água, comparado a solos com cobertura natural, reduzindo a umidade para evaporação e resfriamento (Berdahl; Bretz, 1997; Sobral, 2005). A redução da cobertura verde, o uso intensivo de combustíveis fósseis e a construção de prédios altos, que atuam como barreiras para a ventilação, podem explicar as ilhas urbanas de calor (Sobral, 2005). No Brasil, um estudo mostrou que a diferença média de temperatura entre o centro e a periferia de uma cidade média (cerca de 1,5 milhão de habitantes) foi de $6{ }^{\circ} \mathrm{C}$ (Santos et al., 2013). Já um estudo conduzido na Região Metropolitana do Rio de Janeiro encontrou variabilidades térmicas de até $25^{\circ} \mathrm{C}$ entre áreas com diferentes níveis de urbanização (Sena et al., 2014). As ilhas urbanas de calor podem agravar os períodos prolongados de temperatura muito elevada, eventos conhecidos como ondas de calor.

O aumento da temperatura do ar impacta a flora e a fauna, influenciando a disseminação de doenças causadas por vetores como dengue, zika e febre chicungunya (Coelho-Zanotti; Massad, 2012, Laschewski; Jendritzky, 2002). A taxa de incidência de dengue na cidade de São Paulo (casos por 100 mil habitantes) em 2010-2011 foi maior em áreas que combinavam cobertura vegetal restrita e altas temperaturas médias de superfície $\left(29 \pm 2{ }^{\circ} \mathrm{C}\right)$ do que em áreas com ampla cobertura vegetal: 72,3 casos contra 3,2 casos. A alta temperatura $\left(>32{ }^{\circ} \mathrm{C}\right)$ foi o principal determinante para a concentração dos casos, superando características socioeconômicas como regiões de baixo nível socioeconômico, moradia precária e alta densidade populacional (Araujo et al., 2015).

Eventos de ondas de calor têm sido associados a aumento da mortalidade. $\mathrm{Na}$ Inglaterra e no País de Gales o calor associou-se a aumento da mortalidade de idosos por quase todas as causas no verão no período de 1993 a 2006 (Gasparrini et al., 2012). Um estudo recente, considerando 384 localidades em 13 países, mostrou que temperaturas ambientes desconfortáveis (frio ou calor extremos) foram responsáveis por um aumento de $7,71 \%(95 \%$ CI $7,43-7,91)$ na 
mortalidade. O frio foi o responsável pela maioria das mortes prematuras 7,29\% $(7,02-7,49)$, contra $0,42 \%(0,39-0,44)$ por calor extremo. A grande variabilidade entre os países encontrada no estudo indica uma possível variabilidade da capacidade fisiológica de adaptação a extremos de temperatura das diferentes populações estudadas, mas também, variabilidade na capacidade de adaptação da infraestrutura do país para proteger sua população frente a extremos de temperatura. No Brasil, o frio foi responsável por um aumento de $2,83 \%(2,34-$ $3,30)$ das mortes e o calo,r por 0,70\% (0,45-0,93) (Gasparrini et al., 2015).

Estima-se que a temperatura média no Brasil possa subir entre $2{ }^{\circ} \mathrm{C} \mathrm{e} 4{ }^{\circ} \mathrm{C}$ até 2100, comparado com as médias de temperatura entre 1961 e 1990 (Marengo et al., 2009). No entanto, ao contrário de muitos países desenvolvidos como a Austrália, o Brasil não dispõe atualmente de políticas consistentes para proteger suas populações dos extremos de temperatura.

Modificações no perfil de calor urbano impactam a dispersão de poluentes, a formação de relâmpagos, além de outros processos físicos e químicos do ambiente urbano (Khan; Simpson, 2001). As ondas de calor aumentam a concentração de poluentes atmosféricos nas ilhas de calor urbanas, aumentando a incidência de doenças relacionadas à poluição e já descritas no item "a" deste artigo.

As ilhas urbanas de calor favorecem a formação de um poluente particular, o ozônio troposférico. Formado a partir de nitrogênio e compostos orgânicos voláteis liberados pela queima de combustíveis fósseis, na presença de luz, o ozônio é mais frequente no final da primavera e começo do outono (Hsieh et al., 1999; Andrade et al., 2004). O ozônio troposférico é fortemente relacionado a crises agudas de asma (Andrade et al., 2004), alterações cardiovasculares e inflamação sistêmica, além de aumento da mortalidade (Bell et al., 2014). Entre 1990 e 2013, a mortalidade ajustada por ozônio no mundo aumentou em $19,8 \%$ e a morbidade medida em DALY, em 32,5\% (Forouzanfar et al., 2015). Idosos são o grupo populacional mais vulnerável (Bell et al., 2014).

Em São Paulo, o gradiente térmico entre a zona central e a periférica da cidade pode atingir $10^{\circ} \mathrm{C}$. Embora São Paulo tenha duas estações bem definidas: primavera-verão (quente e úmido) e outono-inverno (frio e seco), eventos extremos de temperatura têm se tornado mais frequentes na cidade, particularmente pela influência das mudanças climáticas (Bornstein; Lin, 2000). No inverno, a qualidade do ar fica frequentemente prejudicada em São Paulo e a população tem que enfrentar os efeitos deletérios da poluição para a saúde humana descritas no item "a" deste artigo. Como a disponibilidade de energia solar é baixa, a dispersão de poluentes depende em grande parte da força da circulação da ilha de calor (Bornstein; Lin, 2000).

No verão, a diferença de temperatura entre o centro (quente) e a periferia (menos quente) da cidade aumenta eventos de chuva forte nas partes centrais da cidade (Freitas et al., 2007), favorecendo enchentes e seus malefícios à saúde como o aumento da incidência de leptospirose. As enchentes também se rela- 
cionam ao estresse emocional devido aos problemas de imobilidade gerados na cidade e à insegurança da integridade dos bens matérias de pessoas que residem em moradias precárias.

A associação das ilhas de calor e das mudanças climáticas na temperatura dos grandes centros urbanos tende a aumentar a frequência e a intensidade de ondas de calor, períodos de seca e de chuva intensa, com impactos negativos para a saúde e bem-estar de quem vive em cidades. Em Shangai, China, um estudo mostrou que as ilhas urbanas de calor foram diretamente responsáveis por excesso de mortalidade devido a condições extremas de temperatura (Tan et al., 2010). Populações de baixo nível socioeconômico são as mais impactadas (Urban et al., 2014; Burkart et al., 2011).

O aumento de áreas verdes nos centros urbanos pode resfriar a temperatura do local e do entorno, mitigando as ilhas urbanas de calor e seus malefícios para a saúde. Além disso, muitos estudos têm associado o aumento da cobertura urbana verde a ganhos para a saúde mental (Roe et al., 2010) e cardiovascular (Villeneuve et al., 2012). Outros benefícios relatados incluem resultados positivos sobre mortalidade (Villeneuve et al., 2012), desfechos de nascimento (Ebisu et al., 2016), recuperação de cirurgia (Ulrich, 1984), dor de cabeça (Hartig et al., 2003) e sono (Astell-Burt et al., 2013). A literatura também descreve benefícios para a saúde social como redução da criminalidade (Wolfe; Mennis 2012) e melhoria nas interações sociais (Mass et al., 2009).

Qual seria o mecanismo por trás dos benefícios para a saúde da exposição a áreas verdes? A explicação mais consistente até o momento é que a proximidade a áreas verdes incentivaria a prática de atividade física. Assim, os benefícios para a saúde viriam da adoção de um estilo de vida menos sedentário, reduzindo o risco de doenças cardiovasculares, obesidade, entre outros benefícios. Num estudo realizado em cidades de oito países europeus, a frequência da prática de atividade física entre os residentes de áreas mais verdes foi três vezes maior do que a frequência de moradores de áreas menos verdes (Ellaway et al., 2005). Nos Estados Unidos, um estudo mostrou que morar a menos de 500 metros de um parque na infância reduz o risco de sobrepeso na adolescência (Wolch et al., 2011). No Brasil, estudos transversais mostraram que regiões com alta densidade de parques e locais públicos para a prática esportiva estão associados a menor prevalência de sobrepeso em São Paulo (Jaime et al., 2011) e obesidade em Belo Horizonte (Matozinhos et al., 2015).

Outros possíveis mecanismos relacionados aos benefícios das áreas verdes para a saúde são a redução da poluição do ar (Janhall, 2015) e da água (Dora et al., 2015), além de redução da poluição sonora (Fan et al., 2010) e vulnerabilidade de habitações ante enchentes (Dora et al., 2015).

Além do aumento das áreas verdes dentro das cidades, o uso de matérias com maior potencial reflexivo na superfície de construções melhora a eficiência energética do prédio e pode auxiliar na mitigação das ilhas urbanos de calor (So- 
lecki et al., 2005). A promoção da agricultura urbana, já discutida no item "b" deste artigo, é uma outra estratégia possível.

\section{d) Conforto térmico e qualidade do ar de ambientes fechados (indoor)}

A poluição do ar em ambientes internos figura entre os principais fatores de risco para doenças crônicas na América Latina (Lim et al., 2012), e foi responsável por 7\% das mortes prematuras na região em 2010 (WHO, 2014). A qualidade do ar em ambientes fechados é modulada por ventilação, níveis de poluentes do ambiente externo e produção de poluentes dentro do ambiente (Rydin et al., 2012). Arquitetos e designers estão na posição de melhorar a ventilação e consequentemente a qualidade do ar interno. A fonte de poluição interna mais estudada é a queima de combustíveis sólidos para cozinhar e aquecer o ambiente interno.

Os impactos para a saúde da poluição em ambientes internos incluem aumento no risco de pneumonia em crianças menores de cinco anos e doença pulmonar obstrutiva crônica, doenças cardiovasculares, catarata e câncer de pulmão em adultos (Smith et al., 2013). Como passam a maior parte do tempo na cozinha ou perto dela, mulheres e crianças pequenas são os mais vulneráveis. A falta de acesso a fontes de energia mais limpas também pode resultar em outros riscos para a saúde como queimaduras, acidentes e intoxicação (Dora et al., 2015).

No Brasil, Fortaleza é a região metropolitana com maior porcentagem de residências sem fogão: 1,76\% (IBGE 2010). No entanto, a porcentagem pode ser significativamente maior nos países menos desenvolvidos da América Latina (Smith et al., 2013; Laborde et al., 2015). Fogões movidos a biomassa mais eficientes e com chaminés (idealmente aqueles que resultam em combustão completa) têm sido uma solução bem-sucedida, como mostrado na queda pela metade da incidência de pneumonia em crianças da Guatemala após a instalação desses fogões (Smith et al. 2011). Esses fogões também reduzem as emissões de gases de efeito estufa, beneficiando a saúde pública e mitigando as mudanças climáticas simultaneamente (Wilkinson et al., 2009). O uso de gás ou eletricidade para cozinhar gera pouca ou nenhuma poluição na cozinha. Assim, eletricidade a preço acessível também resultaria em melhor qualidade do ar interior (Smith et al., 2013).

Outras estratégias para adequar a qualidade do ar interno são aperfeiçoamento na ventilação e iluminação solar das residências, aquecimento interno eficiente e uso de materiais de construção que reduzem a perda de calor pelas paredes, janelas e telhados, entre outras estratégias de redução do uso de energia (Rydin et al., 2012).

Intervenções residenciais que promovam o aquecimento e a eficiência energética do lar podem melhorar a saúde geral dos moradores, particularmente pessoas com doenças respiratórias prévias (Thomson et al., 2013). Ademais, temperaturas interiores baixas representam maior risco para hipertensão arterial. 
Assim, estratégias de conforto térmico tendem a melhorar a saúde cardiovascular (Shiue; Shiue, 2014).

A fumaça do tabaco em ambientes fechados também é uma fonte reconhecida de poluição do ar interna (Laumbach; Kipen, 2014). Essa exposição pode ser eficientemente reduzida mediante políticas de restrição do fumo em ambientes fechados (Sebrié et al., 2008), como já é feito em diversas cidades no mundo e no Brasil.

O amianto é uma substância carcinogênica (IARC, 2009) relacionada a diversas doenças do pulmão (O'Reilly et al., 2007), incluindo, além de câncer, abestose (Bledsoe et al., 2014). Resistente ao fogo e com propriedades isolantes e de absorção do som, o amianto era misturado ao cimento na construção de materiais, especialmente telhados (Haynes, 2010). O amianto foi banido na maioria dos países desenvolvidos (Laborde et al., 2015). Na América Latina, Argentina, Chile, Uruguai e Honduras também baniram a substância (ibidem). Para melhorar a qualidade do ar interior, seria interessante que arquitetos, engenheiros e demais responsáveis por construções onde o amianto ainda não foi banido optassem por materiais sem amianto na sua composição. $\mathrm{O}$ mesmo vale para outros componente tóxicos como o formaldeído (Wang; Zang, 2009).

\section{e) Saneamento}

A Organização das Nações Unidas (ONU) considera o acesso à água segura e ao saneamento direitos humanos básicos (United Nations, 2010). A falta de acesso à água segura para beber, saneamento e/ou higiene afeta gravemente a saúde humana (WHO; Unicef 2014). O principal impacto são surtos de diarreia e consequente aumento da mortalidade infantil. No entanto há uma série de outras doenças reconhecidamente relacionadas como desnutrição, doenças causadas por vetores (exemplo dengue), doenças tropicais negligenciadas (exemplo esquistossomose) e doenças relacionadas a contaminantes químicos presentes na água (Freeman et al., 2013; Bartram et al., 2005). Devido à maior prevalência de falta de acesso a fontes de água potável e ao saneamento em populações de baixa renda, estas são as mais vulneráveis (Dora et al., 2015).

$\mathrm{Na}$ América Latina, o acesso a água potável para beber atingiu $94 \%$ da população em 2012 (WHO; Unicef, 2014). No entanto, mudanças no regime de chuvas, rápido crescimento populacional, alto consumo, falta de planejamento e investimentos, entre outros fatores, desafiam o fornecimento de água potável nos grandes centros urbanos.

A fundação de uma companhia para o fornecimento de água para as residências de São Paulo no final do século XIX foi um marco do início das medidas sanitárias no Brasil. No entanto, o esgoto não recebeu a mesma atenção. A primeira lei para regular a poluição das águas em São Paulo data de 1940. E ainda hoje, a taxa de tratamento do esgoto do estado de São Paulo é de 53,34\% (Sistema Nacional de Informações sobre Saneamento, 2013). Uma possível explicação reside no fato de os investimentos em oferta de água potável serem 
percebidos como prioritários comparados à coleta e tratamento de esgoto, tanto pela população quanto pelos governantes (Rydin et al., 2012).

Esgoto não tratado contribui para acúmulo excessivo de nutrientes nos corpos de água doce (eutrofização), e consequente proliferação de cianobactérias nos reservatórios de água (Cheung et al., 2013). As cianobactérias produzem metabólitos tóxicos para a saúde (ibidem), alguns reconhecidamente carcinogênicos, como a microcistina (IARC 2010). O meio ambiente também é prejudicado (morte de peixes, por exemplo), o que impacta também as atividades turísticas.

Segundo a Organização Mundial da Saúde, uma fonte de água potável segura é aquela protegida de contaminação externa, especialmente contaminação por fezes. Já uma infraestrutura de saneamento adequada é aquela que separa de forma higiênica os excrementos humanos do contato humano como vasos sanitários, sistemas de esgoto canalizado, fossas sépticas, vários tipos de latrinas e banheiros de compostagem (WHO; Unicef; 2014). No entanto a classificação errônea (classificar suprimento de água e esgoto como adequado, quando na verdade não atinge critérios básicos para tal) é frequente (Rydin et al., 2012); e fontes de fornecimento de água consideradas potáveis ainda podem conter contaminação por bactérias ou químicos (Dora et al., 2015).

Além disso, estima-se que os períodos de falta de água nas cidades se tornaram mais frequentes por causa das mudanças climáticas (Dora et al., 2015), ameaçando as conquistas no suprimento de água potável e saneamento alcançadas até o momento. Em 2015, o nível dos reservatórios de água da cidade de São Paulo caiu drasticamente. No começo do ano, a cidade enfrentou uma severa epidemia de dengue. As mudanças no fornecimento de água e o armazenamento inadequado de água da chuva em conjunto podem ter contribuído para o aumento nos casos de dengue em 2015.

Idealmente, todas as residências deveriam ter suprimento de água tratada para banheiros e cozinha, vasos sanitários e sistemas de drenagem de água da chuva para prevenir enchentes. No entanto essa realidade não atinge todos os residentes urbanos, particularmente os moradores de favelas (Rydin et al., 2012).

No Rio de Janeiro, 1,39 milhão de pessoas vivem em favelas. $\mathrm{O}$ acesso a água tratada e saneamento para essas populações é menor, comparado com os demais residentes da cidade (Snyder et al., 2013). O desafio de reduzir essa desigualdade é definir quem vai de fato pagar pela infraestrutura completa. A instalação de vasos sanitários dentro das residências costuma ser responsabilidade individual do morador e não das autoridades governamentais. Famílias de baixa renda, em especial aquelas que moram de aluguel em assentamentos informais, podem ter dificuldades em pagar por essa infraestrutura (Rydin et al., 2012).

\section{Considerações finais}

Os tópicos descritos separadamente neste artigo se sobrepõem sistematicamente aumentando a magnitude dos efeitos para a saúde das exposições 
urbanas. Por exemplo, uma possibilidade de aumentar as áreas verdes discutidas no item "c" deste artigo é a promoção da agricultura urbana com implicações discutidas no item "b". Outra estratégia possível é o aumento do número de parques nas cidades.

Parques estão associados a um aumento no nível de atividade física, o que impacta positivamente a obesidade e outras doenças crônicas. Um estudo conduzido nos Estados Unidos entre 1993 e 1996 mostrou que crianças com acesso facilitado a parques e programas de recreação apresentavam menores índices de obesidade (Wolch et al., 2011). Também nos Estados Unidos, um estudo mostrou que comunidades de baixa renda têm acesso restrito a parques (ibidem). Além disso, os parques costumam ser mais poluídos em áreas de baixa renda (Su et al., 2011). Não é difícil imaginar que a situação no Brasil seja semelhante.

Neste artigo, propusemos uma discussão a partir dos cinco tópicos propostos por Rydin et al. (2012). No entanto, outras intervenções urbanas igualmente moldam a saúde nas cidades e não devem ser ignoradas. Por exemplo, um estudo conduzido por Epstein et al. (2012) mostrou que crianças que moram em bairros com menos lojas de conveniência e supermercados apresentam menor índice de obesidade.

A forma como as cidades funcionam influencia significativamente a saúde de seus moradores. Entender como se dá essa influência é um grande desafio metodológico, em razão das inúmeras variáveis envolvidas num sistema complexo como uma cidade. $\mathrm{O}$ uso de ferramentas metodológicas usadas para estudar sistemas é de grande valia no entendimento dos impactos do ambiente construído para a saúde. As evidências discutidas já oferecem direcionamentos sobre quais exposições presentes nos ambientes urbanos deveriam ser evitadas ou promovidas. Promover efetivamente as mudanças urbanísticas necessárias a uma cidade mais saudável requer a interação multidisciplinar entre diversas pastas do governo, a sociedade civil e outros atores da sociedade. Apesar da magnitude do desafio, o objetivo parece ser forte o suficiente para consensos efetivos, afinal uma cidade que promova a saúde de sua população é interesse de todos.

Ademais, uma cidade saudável deveria ser interesse particular dos profissionais de saúde, pois ela representa um grande auxílio terapêutico no enfrentamento de doenças e promoção da qualidade de vida.

\section{Referências}

AKBARI, H. Energy Saving Potentials and Air Quality Benefits of Urban Heat Island Mitigation. Lawrence Berkeley National Laboratory. 2005. Disponível em: <http:// www.osti.gov/scitech/servlets/purl/860475 >. Acesso em: 8 jun. 2015.

ANALITIS, A. et al. Effects of cold weather on mortality: results from 15 European cities within the PHEWE Project. Am J Epidemiol., v.168, p.1397, 2008. 
ANDERSON, B.G.; BELL, L. Weather-related mortality: how heat, cold, and heat waves affect mortality in the Unitated States. Epidemiology, v.20, n.2, p.205-213, 2009.

ANDRADE, L. H. et al. Mental disorders in megacities: findings from the São Paulo megacity mental health survey, Brazil. PLoS One, v.7, n.2, p.e31879, 2012.

ANDRADE, M. F. et al. Air-quality model simulating photochemical formation of pollutants: the São Paulo Metropolitan Area, Brazil. International Journal Environment and Pollution, v.22, n.4, p.460-75, 2004.

ANGNOTTI, T. Urban agriculture: long-term strategy or impossible dream? Lessons from Prospect Farm in Brooklyn, New York. Public Health, p.1-6, 2015.

ARAUJO R. V et al. São Paulo urban heat islands have a higher incidence of dengue than other urban areas. Braz J Infect Dis, v.19, n.2, p.146-55, 2015.

ASTELL-BURT, T.; FENG, X.; KOLT, G. S. Does access to neighbourhood green space promote a healthy duration of sleep? Novel findings from a cross-sectional study of 259319 Australians. BMJ Open 3, 2013.

BARTRAM, J. et al. Focusing on improved water ans sanitation for health. Lancet, v.365, n.9461, p.819-12, 2005.

BECERRA, J. M. et al. Transport and health: a look at three Latin American cities. Cad. Saúde Pública, v.29, n.4, p.654-66, 2013.

BELL, M. L.; ZANOBETTI, A.; DOMINICI, F. Who is more affected by ozone pollution? A systematic review and meta-analysis. Am J Epidemiol., v.180, n.1, p.15-28, 2014.

BERDAHL, P.; BRETZ, S. Preliminary survey of the solar reflectance of cool roofing materials. Energy and Buildings, v.25, p.149-58, 1997.

BLEDSOE, J. R.; CHRISTIANI, D. C.; KRADIN, R. L. Smoking-associated fibrosis and pulmonary asbestosis. Int J Chron Obstruct Pulmon Dis, v.10, p.31-7, 2014.

BRAGA, A. L. F.; ZANOBETTI, A.; SCHWARTZ, J. The effect of weather on respiratory and cardiovascular deaths in 12 U.S. cities. Environmental Health Perspectives, v.110, n.9, p.859-63, 2002.

BORNSTEIN, R.; LIN, Q. Urban heat islands and summertime convective thunderstorms in Atlanta: three case studies. Atmos Environ., v.34, p.507-16, 2000.

BURKART, K. et al. The effect of atmospheric thermal conditions and urban thermal pollution on all-cause and cardiovascularmortality in Bangladesh. Environ Pollut., v.159, n.8-9, p.2035-43, 2011.

CALDERÓN-GARCIDUEÑAS, L. et al. Long-term air pollution exposure is associated with neuroinflammation, an altered innate immune response, disruption of the blood-brain barrier, ultrafine particulate deposition, and accumulation of amyloid beta- 42 and alpha-synuclein in children and young adults. Toxicol Pathol., v.36, n.2, p.289-310, 2008.

CENTRAL OF DISEASE CONTROL - CDC. Extreme Heat: A Prevention Guide to Promote Your Personal Health and Safety. 2004. Disponível em: <http://www.bt.cdc. gov/disasters/extremeheat/heat_guide.asp>. Acesso em: 8 jun. 2015.

CHETTY, R.; HENDREN, N.; KATZ, L. The Effects of Exposure To Better Neighborhoods on Children: New Evidence from The Moving to Opportunity Experiment. Working Paper 21156. Harvard University, 2015. 
CHEUNG, M. Y.; LIANG, S.; LEE, J. Toxin-producing Cyanobacteria in Freshwater: A Review of the Problems, Impact on Drinking Water Safety, and Efforts for Protecting Public Health. Journal of Microbiology, v.51, n.1, p.1-10, 2013.

CINTRA, M. Os custos dos congestionamentos na cidade de São Paulo. Textos para Discussão da Escola de Economia de São Paulo da Fundação Getulio Vargas FGV-EESP, 2014.

COELHO-ZANOTTI, M. S. S.; MASSAD, E. The impact of climate on Leptospirosis in São Paulo, Brazil. International Journal of Biometeorology, v.56, n.2, p.233-41, 2012.

DORA, C. et al. Indicators linking health and sustainability in the post-2015 development agenda. Lancet, v.385, p.380-91, 2015.

EBISU, K.; HOLFORD, T. R.; BELL, M. L. Association between greenness, urbanicity, and birth weight. Sci Total Environ, v.542, p.750-6, 2016.

ELLAWAY, E.; MACINTYRE, S.; BONNEFOY, X. Graffiti, greenery, and obesity in adults: secondary analysis of European cross sectional survey. BMJ, v.331, p.611-12, 2005.

EPSTEIN, L. H. et al. The built environment moderates effects of family-based childhood obesity treatment over 2 years. Ann Behav Med, v.44, n.2, p.248-58, 2012.

FAN, Y. et al. The investigation of noise attenuation by plants and the corresponding noise-reducing spectrum. J Environ Health, v.72, p.8-15, 2010.

FAO. Growing greener cities in Latin America and the Caribbean: a FAO report on urban and peri-urban agriculture in the region. Rome, 2014.

FOROUZANFAR, M. H.; ALEXANDER, L.; ANDERSON, H. R. Global, regional, and national comparative risk assessmentof 79 behavioural,environmental and occupational, and metabolic risks or clusters of risks in 188 countries,1990-2013: a systematic analysis for the Global Burden of Disease Study 2013. Lancet, 2015. Disponível em: <http://dx.doi.org/10.1016/S0140-6736(15)00128-2>.

FREEMAN, M. C. et al., Integration of water, sanitation, and hygiene for the prevention and control of neglected tropical diseases: a rationale for inter-sectoral collaboration. PLosS Neglected Tropical Disease, v.7, n.9, p.1-9, 2013.

FREITAS, E. D. et al. Interactions of an urban heat island and sea-breeze circulations during winter over the metropolitan area of São Paulo, Brazil. Boundary-Layer Meteorology, v.122, n.1, p.43-65, 2007.

GASPARRINI, A. et al. The effect of high temperatures on cause specific mortality in England and Wales. Occup Environ Med., v.69, n.1, p.56-61, 2012.

GASPARRINI, A. et al. Mortality risk attributable to high and low ambient temperature: a multicountry observational study. Lancet, 2015.

GATTO, N. M. et al. LA sprouts randomized controlled nutrition and gardening program reduces obesity and metabolic risk in latino youth. Obesity, v.23, n.6, p.1244-51, 2015.

GOMEZ, L. F. et al. Urban environment interventions linked to the promotion of physical activity: a mixed methods study applied to the urban context of Latin America. Soc Sci Med, v.131, p.18-30, 2015.

GOUVEIA, N. et al. Hospitalizações por causas respiratórias e cardiovasculares associa- 
das à contaminação atmosférica no Município de São Paulo, Brasil pollution in the city of São Paulo, Brazil. Cadernos de Saúde Pública, v.22, n.12, p.2669-77, 2006.

HAINES, A. et al. Public health benefits of strategies to reduce greenhouse-gas emissions: overview and implications for policymakers. Lancet, v.374, n.9707, p.2104-14, 2009.

HARA, Y. et al. Quantitative assessment of the Japanese "local production for local consumption" movement: a case study of growth of vegetables in the Osaka city region. Sustain Sci., v.8, p.515-27, 2013.

HARTIG, T. et al. Tracking restoration in natural and urban field settings. J. Environ. Psychol., v.23, p.109-23, 2003.

HAYNES, R. C. Aworn-out welcome: renewed call for a global ban on absbestos. Environmental Health Perspectives, v.118, n.7, p.298-303, 2010.

HEI. Panel on the Health Effects of Traffic-Related Air Pollution. Traffic-Related Air Pollution: A Critical Review of the Literature on Emissions, Exposure, and Health Effects. HEI Special Report 17. Health Effects Institute, Boston, MA 2010. Diponível em: <http://pubs.healtheffects.org/getfile.php?u=553>.

HSIEH, C.; CHANG, K.; KAO, Y. Estimating the ozone formation potential of volatile aromatic compounds in vehicle tunnels. Chemosphere, v.39, p.1433-44, 1999.

HU, F. B.; MANSON, J. E.; WILLETT, W. C. Types of dietary fat and risk of coronary heart disease: a critical review. J Am Coll Nutr., v.20, n.1, p.5-19, 2011.

IARC - International Agency for Research on Cancer. A review of human carcinogens. Part C: Arsenic, metals, fibres, and dusts - IARC Working Group on the Evaluation of Carcinogenic Risks to Humans. Lyon, France: International Agency for Research on Cancer, 2009. Disponível em: <http://monographs.iarc.fr/ENG/Monographs/ voll00C/monol00C.pdf>. Acesso em: 30 maio 2015.

World Health Organization International Agency For Research On Cancer. Monographs on the Evaluation of Carcinogenic Risks to Humans: Ingested Nitrate and Nitrite, and Cyanobacterial Peptide Toxins. v.94, 2010. Disponível em: <http:// monographs.iarc.fr/ENG/Monographs/vol94/mono94.pdf>.

JAIME, P. C. et al. Investigating environmental determinants of diet, physical activity, and overweight among adults in Sao Paulo, Brazil. J Urban Health, v.88, n.3, p.567$81,2011$.

JANHALL, S. Review on urban vegetation and particle air pollution e Deposition and dispersion. Atmos Environ, v.105, p.130-7, 2015.

KESSLER R. Urban gardening: managing the risks of contaminated soil. Environ $\mathrm{He}$ alth Perspect, v.121, n.11 e 12, p.326-33, 2013.

KHAN, S. M.; SIMPSON, R. W. Effect of a heat island on the meteorology of complex urban airshed. Boundary-Layer Meteorol., v.100, p.487-506, 2001.

LABORDE, A. et al. Children's Health in Latin America: The Influence of Environmental Exposures. Environmental Health Perspectives, v.123, n.3, p.201-10, 2015.

LASCHEWSKI, G.; JENDRITZKY. G. Effects of the thermal environment on human health: an investigation of 30 years of daily mortality data from SW Germany. Climate Research, v.21, p.91-103, 2002. 
LAUMBACH, R.; KIPEN, H. Mechanistic data support protecting non-smokers from the lethal effects of second-hand smoke. International Journal of Public Health, v.59, n.4, p.575-6, 2014.

LAVERTY, A. A. et al. Associations between active travel and weight, blood pressure and diabetes in six middle income countries: a cross-sectional study in older adults. Int J Behav Nutr Phys Act, v.20, p.12-65, May 2015.

LIM, S. S. et al. A comparative risk assessment of burden of disease and injury attributable to 67 risk factors and risk factor clusters in 21 regions, 1990-2010: a systematic analysis for the Global Burden of Disease Study 2010. Lancet, v.380, p.2224-60, 2012. LOOMIS, D. et al. International Agency for Research on Cancer Monograph Working Group IARC. The carcinogenicity of outdoor air pollution. Lancet Oncol, v.14, n.13, p.1262-3, 2013.

MAAS, J. et al. Morbidity is related to a green living environment. J Epidemiol Community Health, v.63, p.967-73, 2009.

MARENGO, J. A. et al. Future change of temperature and precipitation extremes in South America as derived from the PRECIS regional climate modeling system. International Journal of Climatology, v.29, n.15, p.2241-55, 2009.

MATOZINHOS, F. P. et al. Neighbourhood environments and obesity among adults: A multilevel analysis of an urban Brazilian context. Prev Med Rep, v.29, n.2, p.337-41, 2015 .

MCMICHAEL, A. J. Transport and health: assessing the risks. In: FLETCHER, T.; MCMICHAEL, A. J. (Ed.) Health at the crossroads: transport policy and urban health Chichester: John Wiley \& Sons, 1996. p.9-26.

MIDDLE, I. et al. Integrating community gardens into public parks: an innovative approach for providing ecosystem services in urban areas. Urban Forestry \& Urban Greening, v.13, p.638-45, 2014.

MILBOURN, L. S.; VAIL, B.A. Sowing the seeds of success: cultivating a future for community gardens. Landscape Journal, v.29, p.1-10, 2010.

MUELLER, N. et al. Health impact assessment of active transportation: A systematic review. Prev Med, v.18, n.6, p.103-14, 2015.

NAWROT, T. S. et al. Public health importance of triggers of myocardial infarction: a comparative risk assessment. Lancet, v.26, n.377(9767), p.732-40, 2011.

NEWMAN, P.; KENWORTHY, G. Cities and sustainability: reducing auto dependency. Washington, DC: Island Press, 1999.

O'REILLY, K. M. A. et al. Asbestos-related lung disease. American Family Physician, v.75, n.5, p.683-8, 2007.

OLLILA, E. Health in All Policies: From rhetoric to action. Scandinavian Journal of Public Health, v.39, n.6, p.11-18, 2011.

PUCHER, J.; BUEHLER, R. At the frontiers of cycling: policy innovations in the Netherlands, Denmark, and Germany. World Transport Policy \& Practice, v.13, p.9-56, 2007.

RISSEL, C. E. Active travel: a climate change mitigation strategy with co-benefits for health. NSW Public Heal. Bull., p.20-13, 2009. 
ROE, J. J. et al. Green space and stress: Evidence from cortisol measures in deprived urban communities. Int J Environ Res Public Health, v.10, p.4086-4103, 2010.

RYDIN, Y. et al. Shaping cities for health: complexity and the planning of urban environments in the 21st century. Lancet, v.379, n.9831, p.2079-2108, 2012.

SALDIVA, P. H. N.; KING, H.; DELMONTEETAL, V. L. C. Respiratory alterations due to urban air pollution: an experimental study in rats. Environmental Research, v.57, n.1, p.19-33, 1992.

SANTOS, T. O. et al. Influence of urbanization on land surface temperature in Recife city. Eng Agric, v.33, n.6, p.1234-44, 2013.

SEBRIÉ, E. M.; SCHOJ, V.; GLANTZ, S. A. Smoke free environments in Latin America: on the road to real change? Prevention and Control, v.3, p.21-35, 2008.

SENA, C. A. P.; FRANCA, J. R. de A.; PERES, L. F. Estudo da Ilha de Calor na Região Metropolitana do Rio de Janeiro Usando Dados do MODIS. Anu. Inst. Geocienc. [online], v.37, n.2, p.111-22, 2014.

SHIUE, I.; SHIUE, M. Indoor temperature below $18{ }^{\circ} \mathrm{C}$ accounts for $9 \%$ population attributable risk for high blood pressure in Scotland. International Journal of Cardiology, v.171, p.el-e2, 2014.

SILVA DIAS, M. M. F. et al. Changes in extreme daily rainfall for São Paulo, Brazil. Climatic Change, v.116, n.3-4, p.705-22, 2013.

SISTEMA NACIONAL DE INFORMAÇÃO SOBRE SANEAMENTO-SIS. 2013. Disponível em: <http://www.snis.gov.br/PaginaCarrega.php?EWRErterterTERTer=105>. Acesso em: 15 ago. 2015.

SMITH, K. R. et al. Effect of reduction in household air pollution on childhood pneumonia in Guatemala (RESPIRE): a randomised controlled trial. Lancet, v. 378, p.171726, 2011 .

SMITH, K. R. et al. Energy and Human Health. Annu. Rev. Public Health, v.34, p.159$88,2013$.

SNYDER, R. E. et al. A comparison of social and spatial determinants of health between formal and informal settlements in a large metropolitan setting in Brazil. Journal of Urban Health: Bulletin of the New York Academy of Medicine, v. 91, n.3, p.432-55, 2013.

SOBRAL, R. H. Heat Island in São Paulo, Brazil. Critical Public Health, v.15, n.2, p.147-56, 2005.

SOLECKI, W. D. et al. Mitigation of the heat island effect in urban New Jersey. Global Environmental Change Part B: Environmental Hazards, v.6, n.1, p.39-49, 2005.

SOUZA, E. L. L.; MACEDO, I. Etanol e bioeletricidade: A cana-de-açucar no futuro da matriz energética. UNICA - União da Indústria de Cana-de-açúcar, 2010.

STIEB, D. M. Ambient air pollution, birth weight and preterm birth: A systematic review and meta-analysis. Environmental Research, v.117, p.100-11, 2012.

$\mathrm{SU}, \mathrm{J}$. G. et al. Does exposure to air pollution in urban parks have socioeconomic, racial or ethnic gradients? Environmental Research, v.111, p.319-28, 2011.

SWINTON, S. M. et al. Ecosystem services and agriculture: Cultivating agricultural ecosystems for diverse benefits. Ecological Economics, v.64, 2007. 
TAN, J. et al. The urban heat island and its impact on heat waves and human health in Shanghai. Int J Biometeorol, v.54, p.75-84, 2010.

THOMSON, H. et al. Housing improvements for health and associated socioeconomic outcomes (Review). Cochrane Database Syst Rev. 2 (CD008657), v.1, p.333, 2013.

TOHILL, B. C. et al. What epidemiologica studies tell us about the relationship between fruit and vegetable consumption and body weight. Nutrition Reviews, v.62, n.10, p.365-74, 2004.

TORREGGIANI, D.; DALL'ARA, E.; TASSINARI, P. The urban nature of agriculture: Bidirectional trends between city and countryside. Cities, v.29, p.412-16, 2012.

ULRICH, R. S. Views through a Windows may influence recovery from surgery. Science, v.224, p.420-1, 1984.

UNITED NATIONS. General Assembly, Resolution 62/292, from july, 21st, 2010. Disponível em http://www.un.org/es/comun/docs/?symbol=A/RES/64/ 292\&lang=E $>$. Acesso em: 15 mar. 2016.

URBAN, A.; DAVÍDKOVOVÁ, H.; KYSELÝ, J. Heat- and cold-stress effects on cardiovascular mortality and morbidity among urban and rural populations in the Czech Republic. Int J Biometeorol, v.58, n.6, p.1057-68, 2014.

VILLENEUVE, P. J. et al. A cohort study relating urban green space with mortality in Ontario, Canada. Environ Res, v.115, p.51-8, 2012.

WANG, X.; ZHANG, Y. A new method for determining the initial mobile formaldehyde concentrations, partition coefficients, ans diffusion coefficients of dry building material. Journal of the Air \& Waste Management Association,v.69, n.7, p.819-25, 2009.

WATTS, N. et al Health and climate change: policy responses to protect public health. Lancet. Published Online: 22 June 2015.

WHAT Epidemiologic Studies Tell Us about the Relationship between Fruit and Vegetable Consumption and Body Weight. Nutrition Reviews, v.62, n.10, p.365-74, 2004.

WILKINSON, P. et al. Public health benefits of strategies to reduce greenhouse-gas emissions: household energy. Lancet, v.374, p.1917-29, 2009.

WOLCH, J. R.; BYRNE, J.; NEWELL, J. P. Urban green space, public health, and environmental justice: The challenge of making cities 'just green enough'. Landscape and Urban Planning, v.125, p.234-44, 2014.

WOLCH, J. R. et al. Childhood obesity and proximity to urban parks and recreational resources: a longitudinal cohort study. Health Place, v.17, p.207-14, 2011.

WOLFE, M. K.; MENNIS, J. J. Does vegetation encourage or suppress urban crime? Evidence from Philadelphia, PA. Landscape Urban Plann, v.108, p.112-22, 2012.

WORLD HEALTH ORGANIZATION. Health in the green economy: health co-benefits of climate change mitigation - the transport sector. 2011. Disponível em: <http://www.who.int/hia/examples/trspt_comms/hge_transport_lowresdurban_30_11_2011.pdf>. Acesso em: 15 de jan. 2014.

WORLD HEALTH ORGANIZATION and UNICEF - WHO/UNICEF. Progress on Drinking Water and Sanitation - update 2014. Switzerland, 2014. Disponível em: <http://www.who.int/water_sanitation_health/publications/2014/jmp-report/ en/>. Acesso em: 2 maio 2015. 
ZEZZA, A.; TASCIOTTI, L. Urban agriculture, poverty, and food security: Empirical evidence from a sample of developing countries. Food Policy, v.35, p.265-73, 2010.

RESUMO - Morar próximo a um parque pode reduzir o risco de infarto do miocárdio, enquanto a exposição à poluição do ar ambiente aumenta o risco. Evidências associam a exposição crônica ao ruído do tráfego ao ganho de peso. Em 2014, mais de mil pessoas morreram no trânsito de São Paulo. No entanto, recomendar que as pessoas fiquem em casa com as janelas fechadas é inviável. A adoção de hábitos saudáveis é favorecida ou dificultada pelo ambiente construído e pelo modo como a cidade está organizada. Além disso, alguns fatores de risco para doenças, como a exposição à poluição do ar, vão além de escolhas individuais e dependem, em grande parte, de ações governamentais. Neste artigo discutimos os aspectos que podem favorecer ou dificultar a promoção da saúde nas cidades, a partir dos tópicos propostos por Rydin et al. (2012).

Palavras-Chave: Saúde, Cidade, Ambiente construído, Políticas públicas.

ABSTRACT - Living near a park can reduce the risk for myocardial infarction, while exposure to outdoor air pollution increases it. There is evidence associating chronic exposure to traffic noise and weight gain. In 2014, more than one thousand people died in the São Paulo's traffic. However, recommending that people stay home with the windows closed is unfeasible. The adoption of healthy habits is aided or hindered by the man-made environment and by how the city is organized. Moreover, some risk factors for disease, such as exposure to air pollution, are beyond individual choices and call for governmental actions. In this paper, we discuss features that may aid or hinder the promotion of health in the city, based on a framework proposed by Rydin et al. (2012).

KErWORDs: Health, City, Man-made environment, Public policies.

Lais Fajersztajn é pesquisadora e doutorada no Laboratório de Poluição Atmosférica Experimental da Faculdade de Medicina da Universidade de São Paulo (LIM 05); graduada em Fisioterapia e especialista em reabilitação gerontológica.

@ - laisfajer@gmail.com

Mariana Veras é bióloga, doutora em Fisiopatologia e especialista em Políticas Públicas e Planejamento Estratégico pela UnB. Atua como pesquisadora científica do Estado de São Paulo, junto ao Hospital das Clínicas - LIM 05, no Departamento de Patologia da Universidade de São Paulo. @-verasine@usp.br

Paulo Hilário Nascimento Saldiva é médico patologista, professor titular do Departamento de Patologia da Faculdade de Medicina da Universidade de São Paulo e diretor do Instituto de Estudos Avançados da Universidade de São Paulo (2016-2020). @ - pepino@usp.br

Recebido em 2.3.2016 e aceito em 15.3.2016.

I, III Faculdade de Medicina,Universidade de São Paulo. São Paulo/São Paulo, Brasil.

${ }^{\text {II }}$ Hospital das Clínicas, São Paulo/São Paulo, Brasil. 